\title{
Operational experience with the ATLAS Pixel Detector at the LHC
}

Cécile Lapoire (University of Bonn, Germany)

On behalf of the ATLAS collaboration

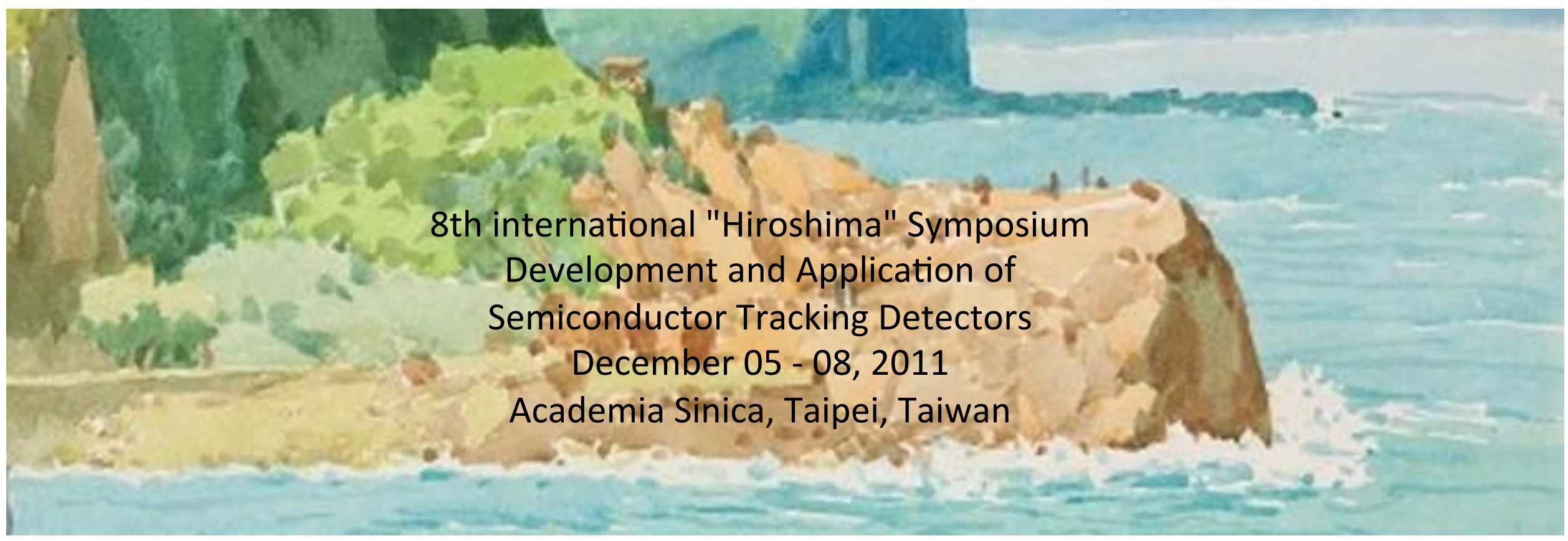




\section{Outline}

- ATLAS

- Overview

- Pixel Detector

- Calibration, tuning and performance

- Calibration overview

- Optolink tuning

- Module tuning (threshold, ToT)

- Noise

- Hit-to-track association efficiency

- Damages

- Non-working modules

- Radiation damages

- Leakage current

- Depletion voltage

- Summary and current status of the Pixel Detector 


\section{ATLAS}

- 44 m long

- $25 \mathrm{~m}$ high

- 7000 tons
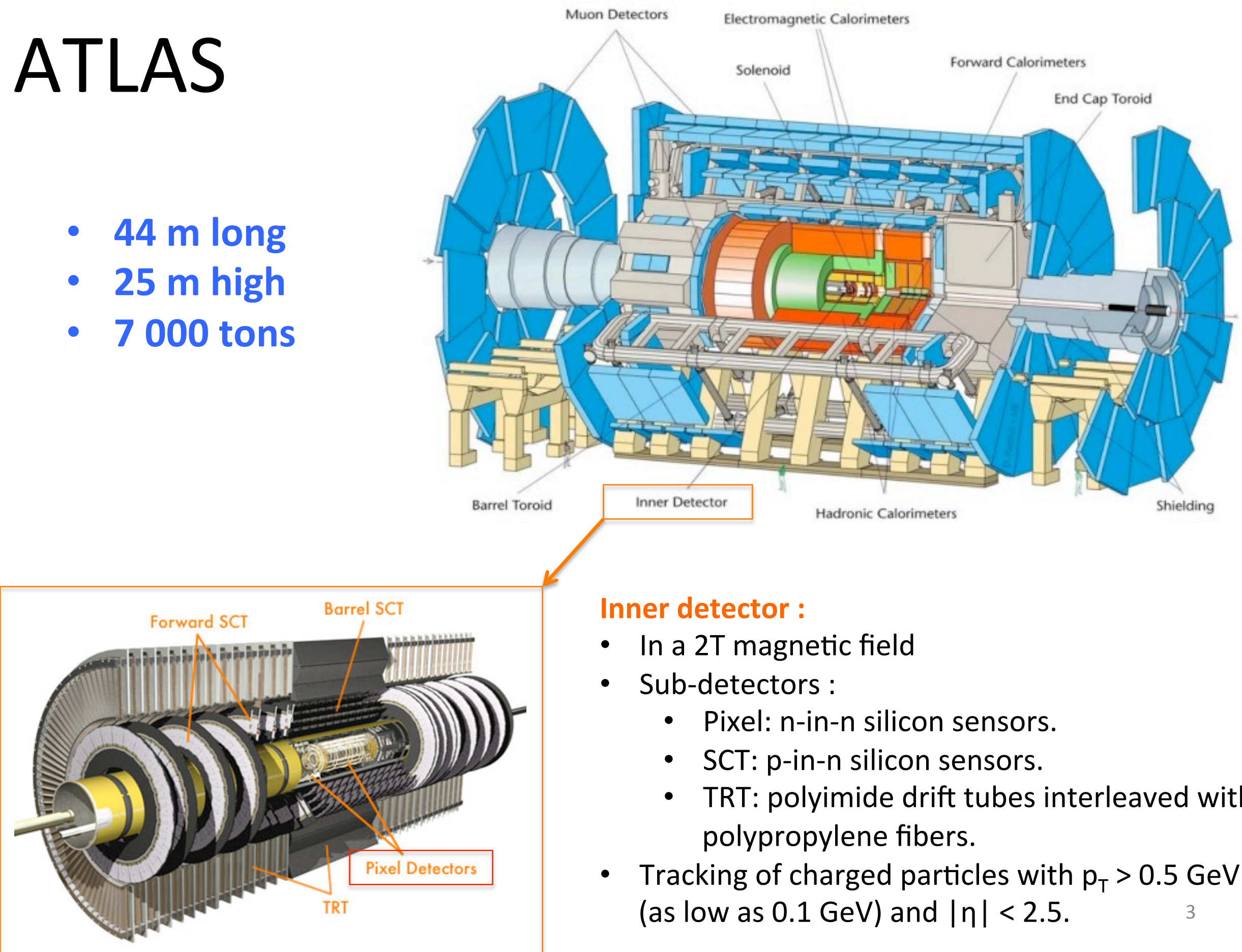

Inner detector:

- In a 2T magnetic field

- Sub-detectors :

- Pixel: $n-i n-n$ silicon sensors.

- SCT: p-in-n silicon sensors.

- TRT: polyimide drift tubes interleaved with polypropylene fibers.

- Tracking of charged particles with $p_{\mathrm{T}}>0.5 \mathrm{GeV}$ (as low as $0.1 \mathrm{GeV}$ ) and $|\eta|<2.5$. 


\section{Pixel Detector : overview}

- 1744 modules

- 1456 on the 3 barrel layers (13 per stave) $\rightarrow$

- 288 on the 6 disks ( 3 on each side)

- Cooling system shared with SCT

- $\mathrm{C}_{3} \mathrm{~F}_{8}$ circulating in support structure
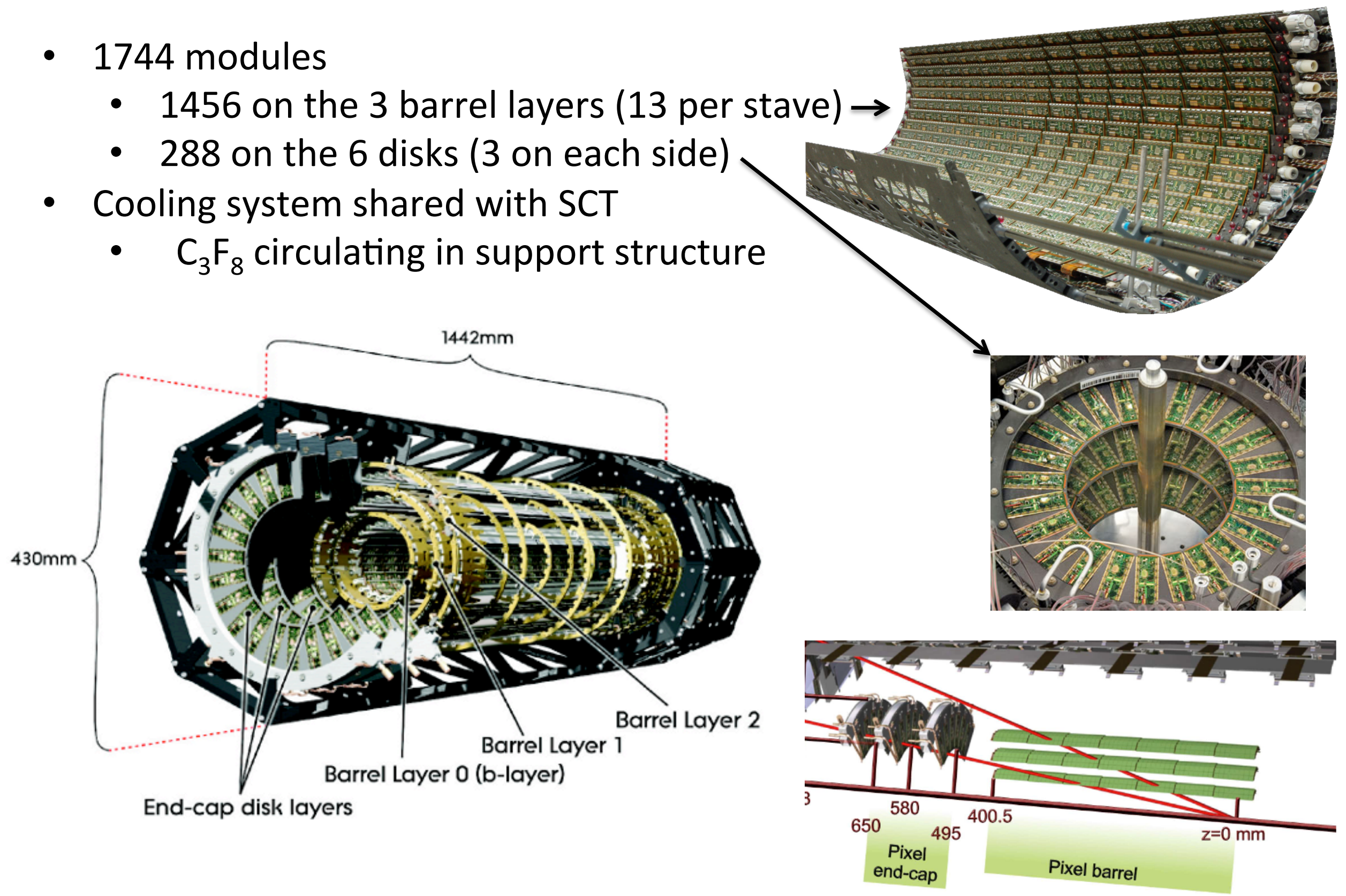


\section{Pixel Detector}

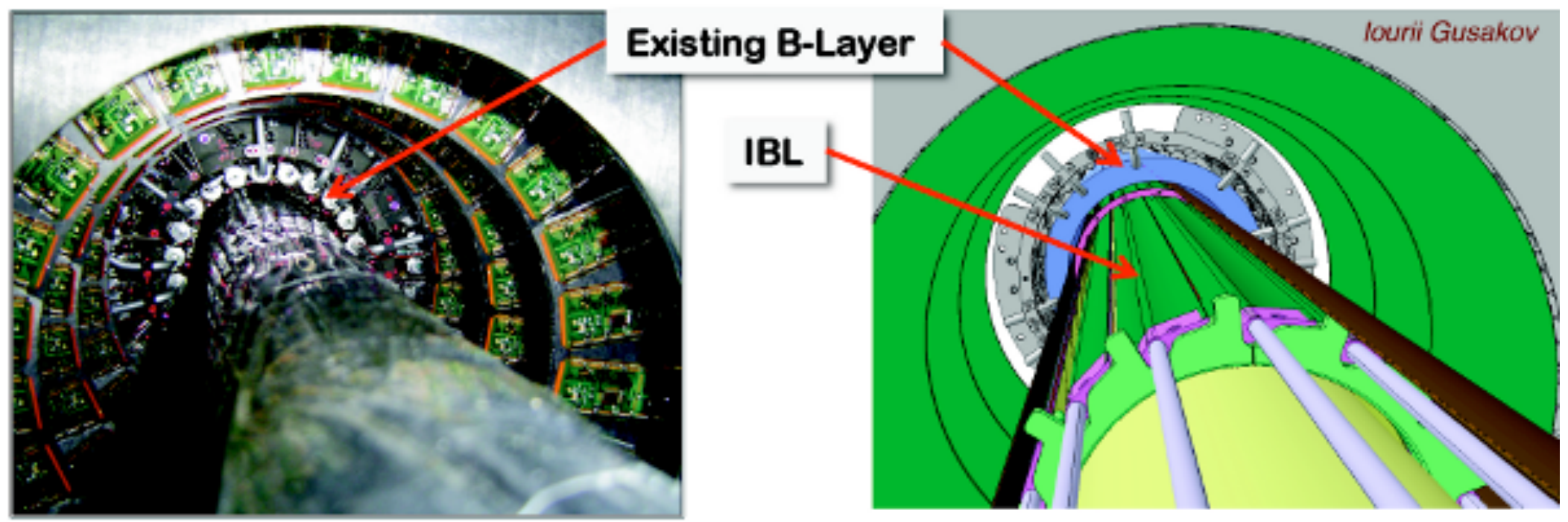

- Innermost layer at $5.05 \mathrm{~cm}$ from the beam axis

- IBL (Inserted b-layer) :

- At $3.3 \mathrm{~cm}$ from the beam axis

- Expected implementation : 2013

- Starting to take data : 2014 


\section{Pixel Detector : modules}

- $250 \mu \mathrm{m}$ thick silicon sensor with an active area of $16.4 \times 60.8 \mathrm{~mm}^{2}$

- 16 Front-ends per module bump bonded to the sensor

- $>80 \mathrm{M}$ readout channels (pixels)

- Resolution

- $10 \mu \mathrm{m}$ in $\mathrm{R} \phi$

- $110 \mu \mathrm{m}$ in z

- Modules operating at $\sim 13^{\circ} \mathrm{C}$ (warmest module at $-5{ }^{\circ} \mathrm{C}$ )

- Modules tilted by $20^{\circ}$ in $\mathrm{R} \phi$ plane
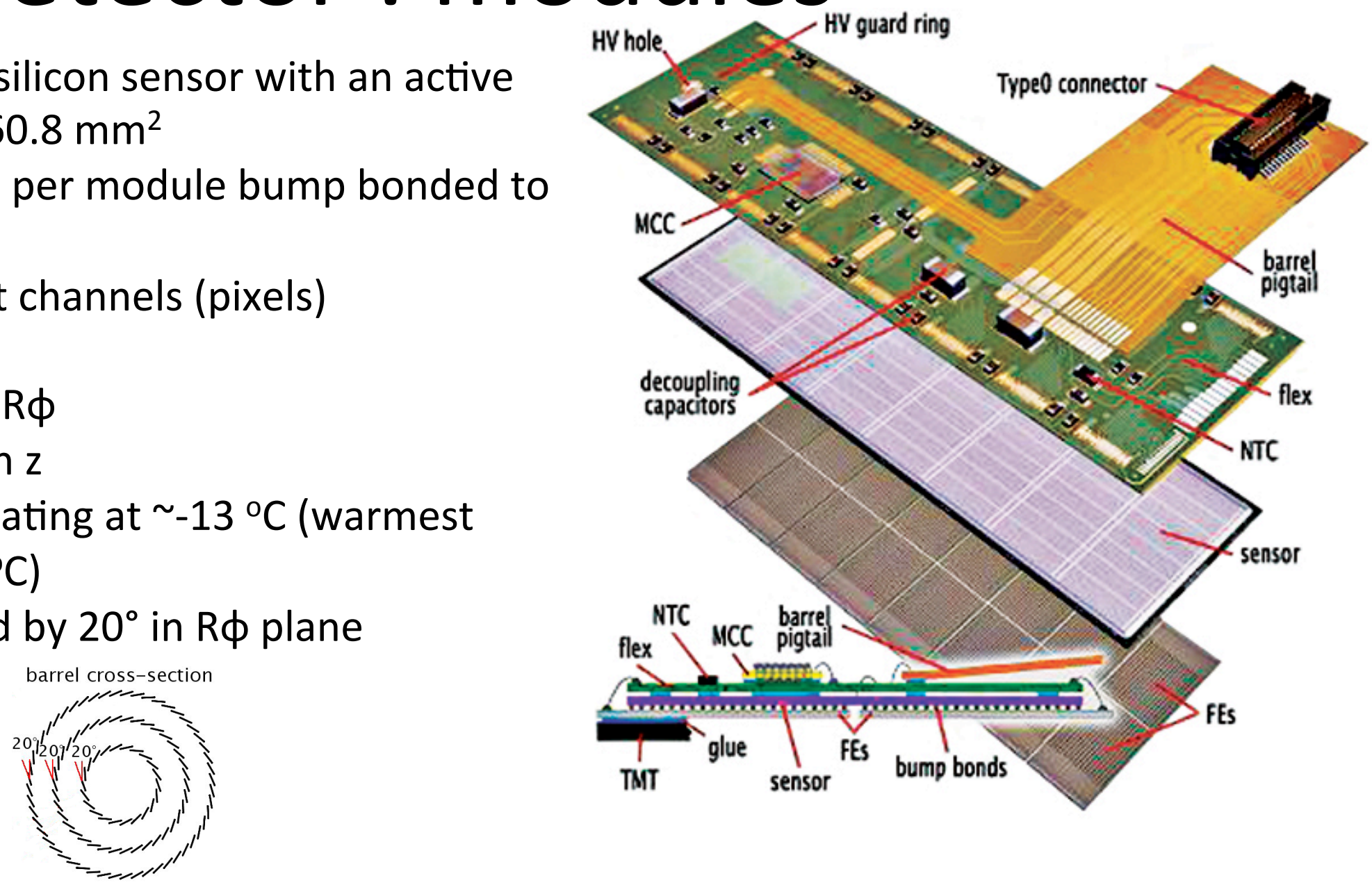

- Module controller chip (MCC) to send trigger, commands and configuration data, and to perform event building.

- $2 \times 80 \mathrm{Mb} / \mathrm{s}$ data transfer speed in b-layer

- $80 \mathrm{Mb} / \mathrm{s}$ in layer 1 and disks

- $40 \mathrm{Mb} / \mathrm{s}$ in layer 2 


\section{Pixel Detector : pixels}

- 47232 (328x144) pixels/module, 46080 readout channels bump-bonded to 16 FE chips

- Special pixels with different size/capacitance to cover inter FE chips

- Sorted with increasing capacitance (noise) : normal, inter-ganged, long, inter long-ganged, ganged, long-ganged,
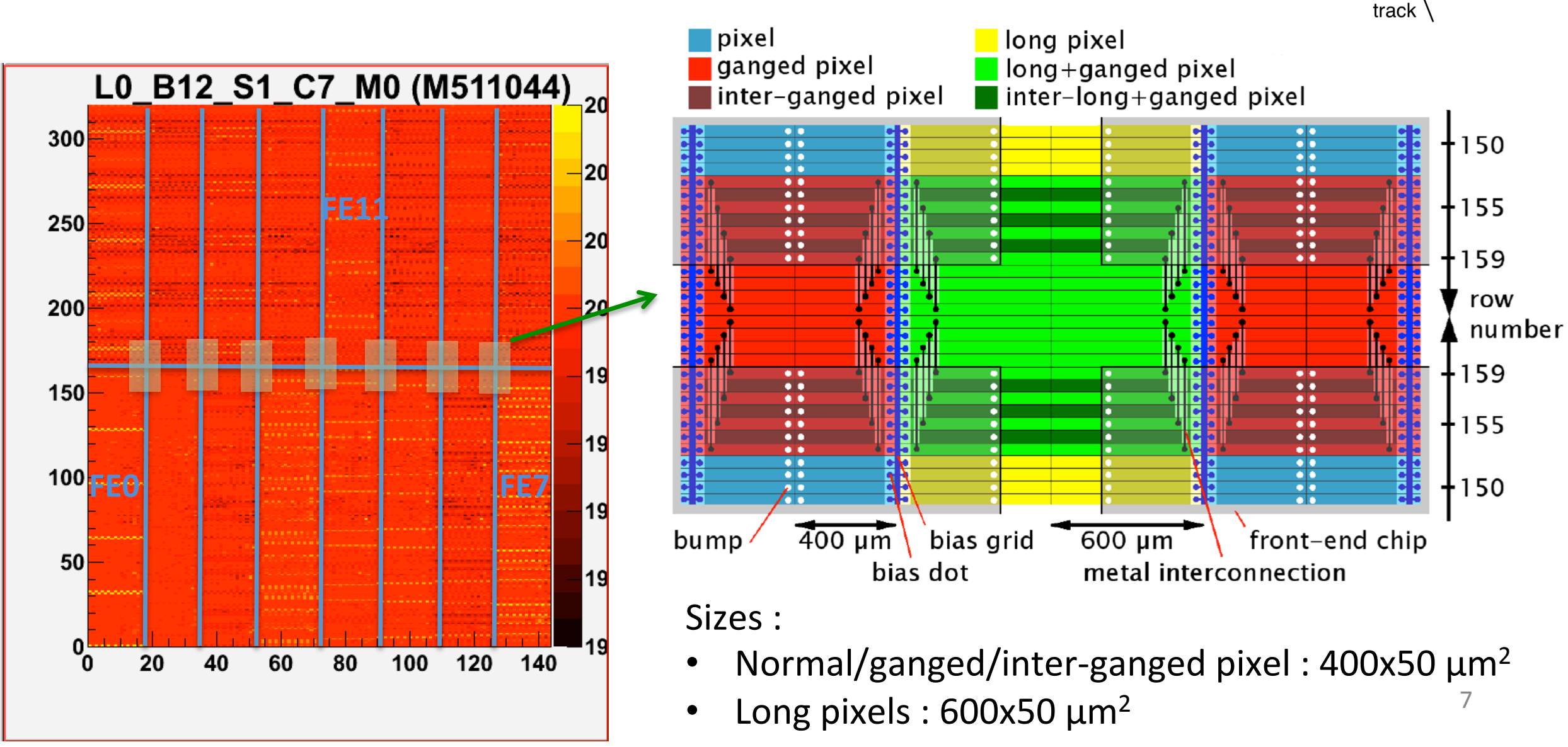

Sizes :

- Normal/ganged/inter-ganged pixel : $400 \times 50 \mu \mathrm{m}^{2}$

- Long pixels : $600 \times 50 \mu \mathrm{m}^{2}$ 


\section{Calibrations}

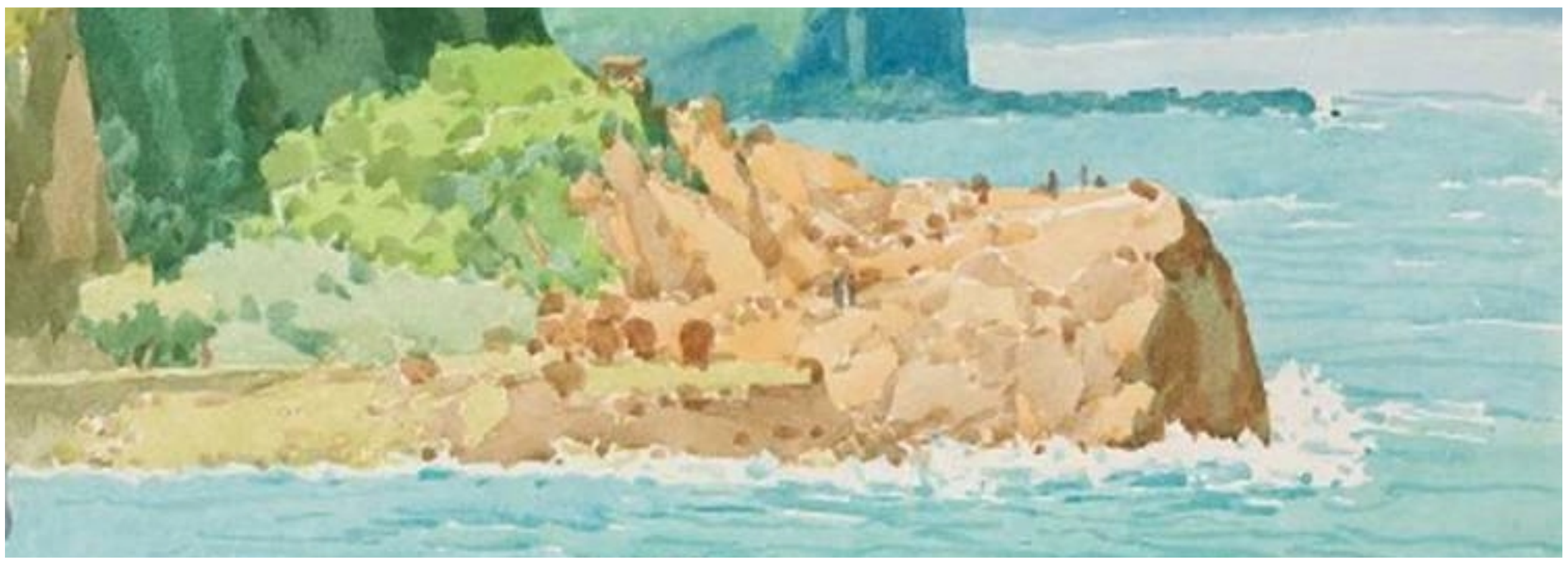




\section{Calibrations}

- Complete calibration process

- Performed during each technical stop on the whole detector

- New noise mask is created

- Takes 3-4 days of $\sim 12$ hours shifts

- After dead Txs replacement

- Corresponding modules timing is adjusted, optolink and modules are checked

- 4 hours

- After power cuts

- Optolinks and modules are checked

- $\sim 4$ hours

- During calibration time during normal data taking periods

- Modules throwing errors are retuned

- 1 hour
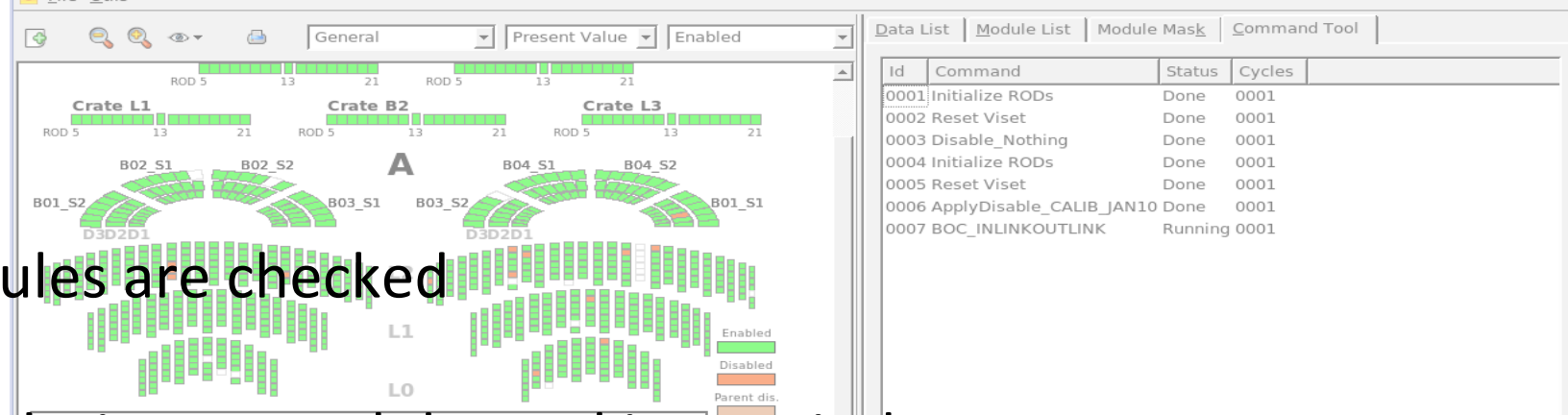

- 1hour

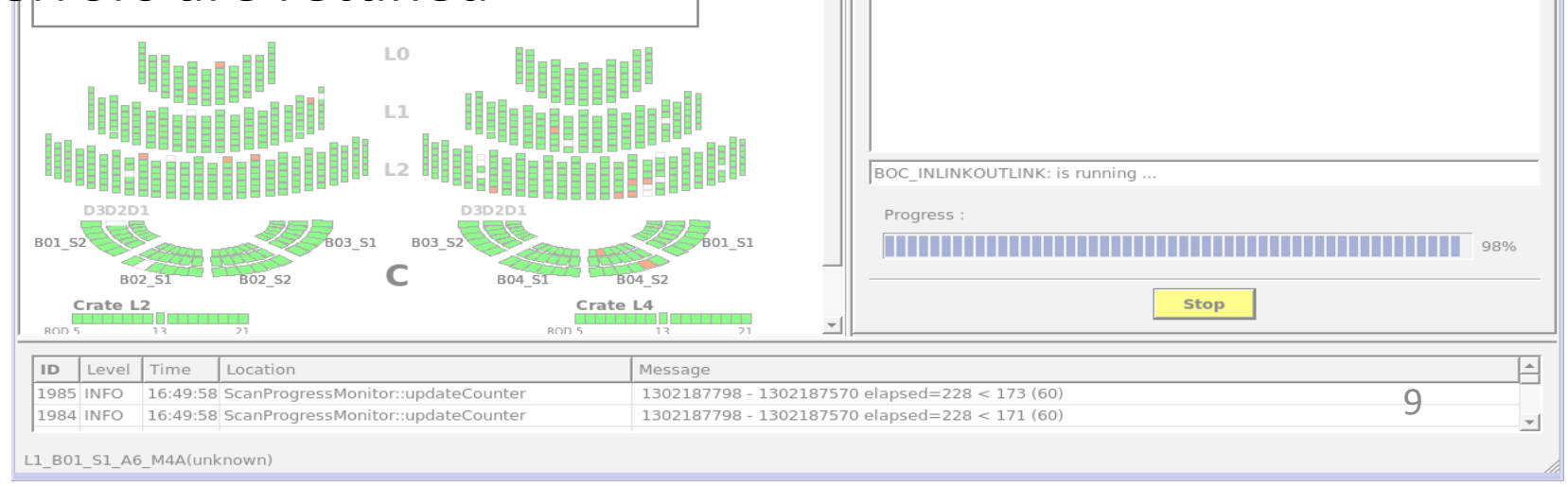




\section{Optolink calibration}

Tuned values to avoid readout errors :

- Off-detector sampling threshold vs phase :

- On-detector laser power (Viset)

- 1 per optoboard (=6 or 7 modules) :
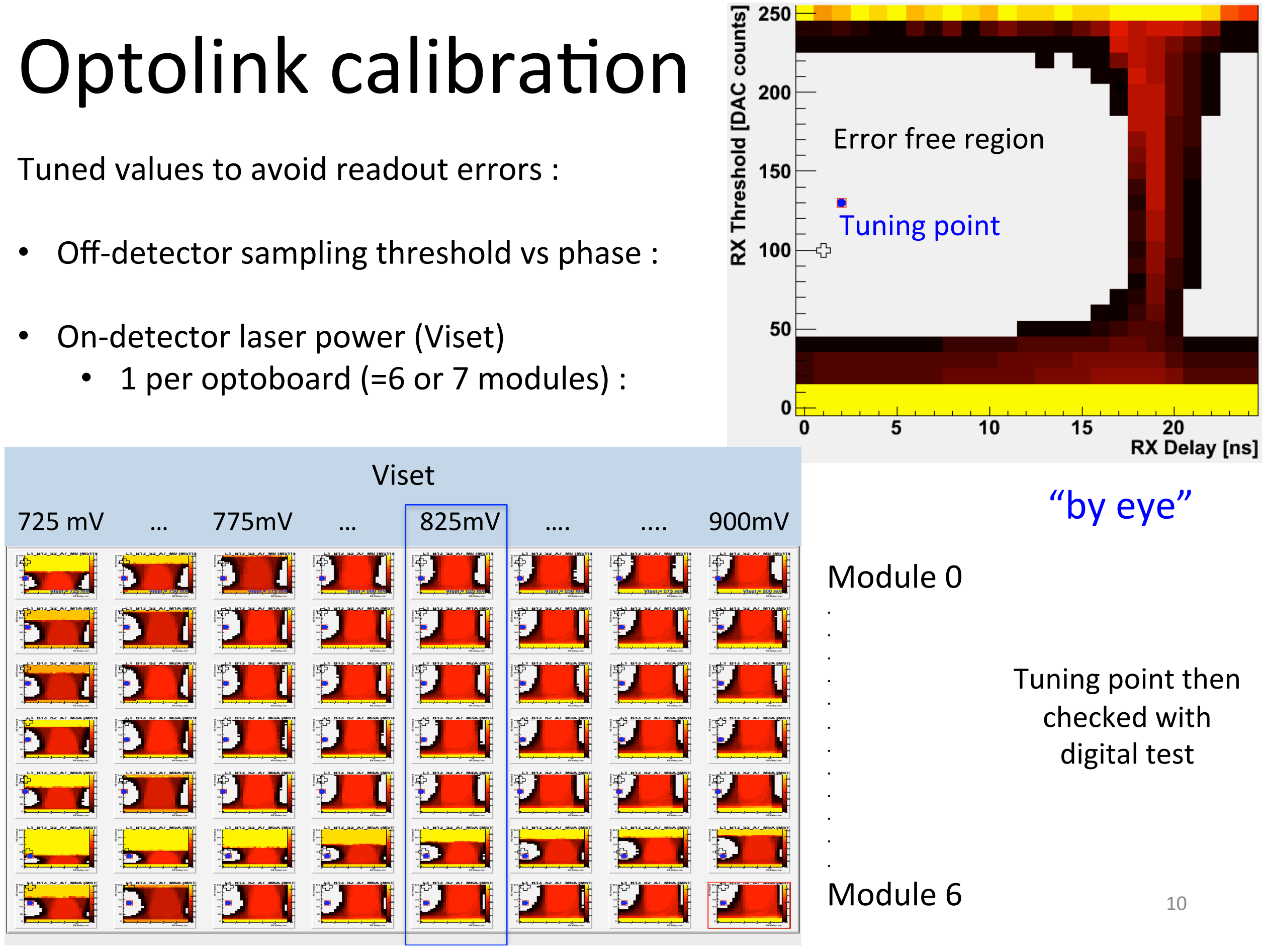


\section{Module calibration}

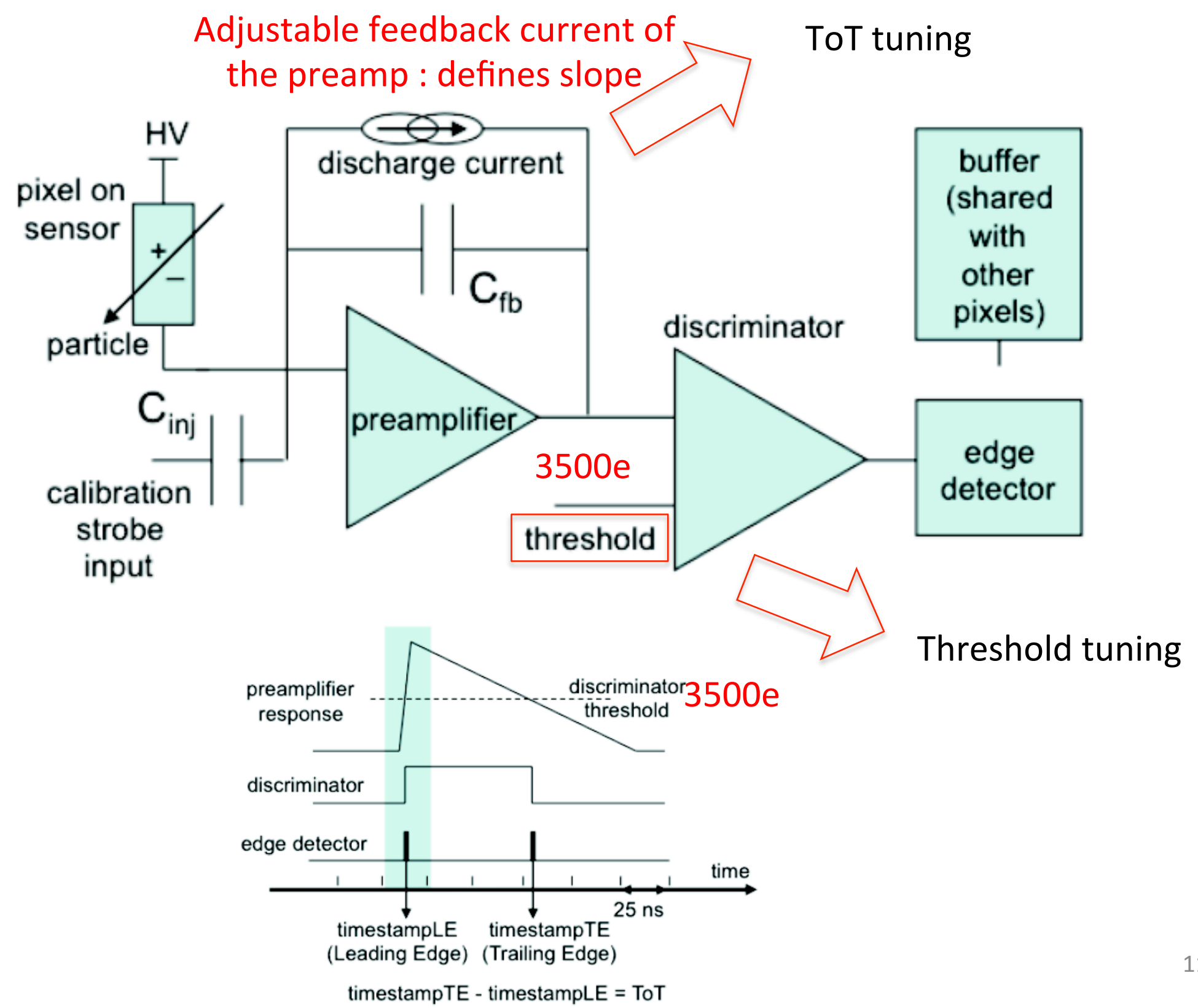




\section{Threshold calibration}

- Tuned at $3500 \mathrm{e}$

- 40 e dispersion after tuning

- Pixel noise with this tuning :

- Normal : 170 e

- Long : 200 e

- Ganged : 300 e

- Successful tests performed at the limit threshold of $2400 \mathrm{e}$

- $\rightarrow$ we are confident for the next

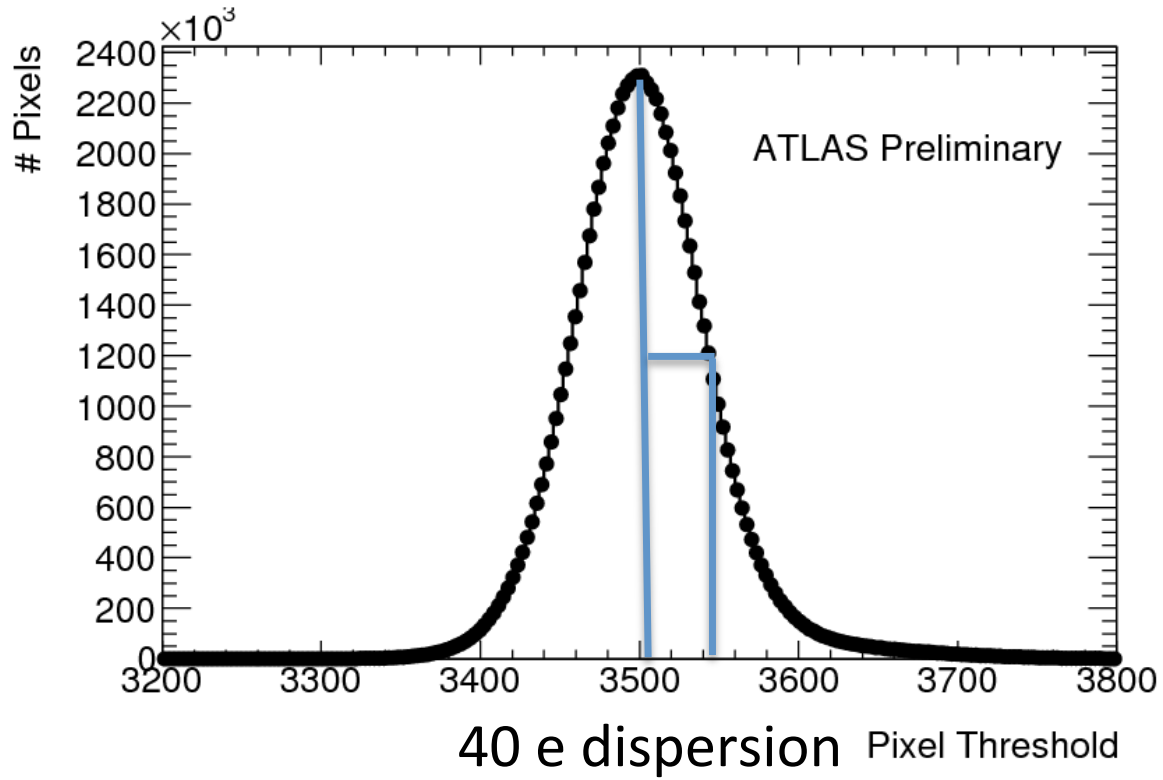
target : tuning at $3000 \mathrm{e}$

\section{Procedure :}

- Inject \# of test charges at target threshold, vary 7-bit DAC per pixel and count generated hits. Optimal value is at $50 \%$ efficiency.

- If 7-bit DAC per pixel does not produce sufficient range of thresholds for tuning target, use 5-bit DAC per FE first to shift global threshold per FE.

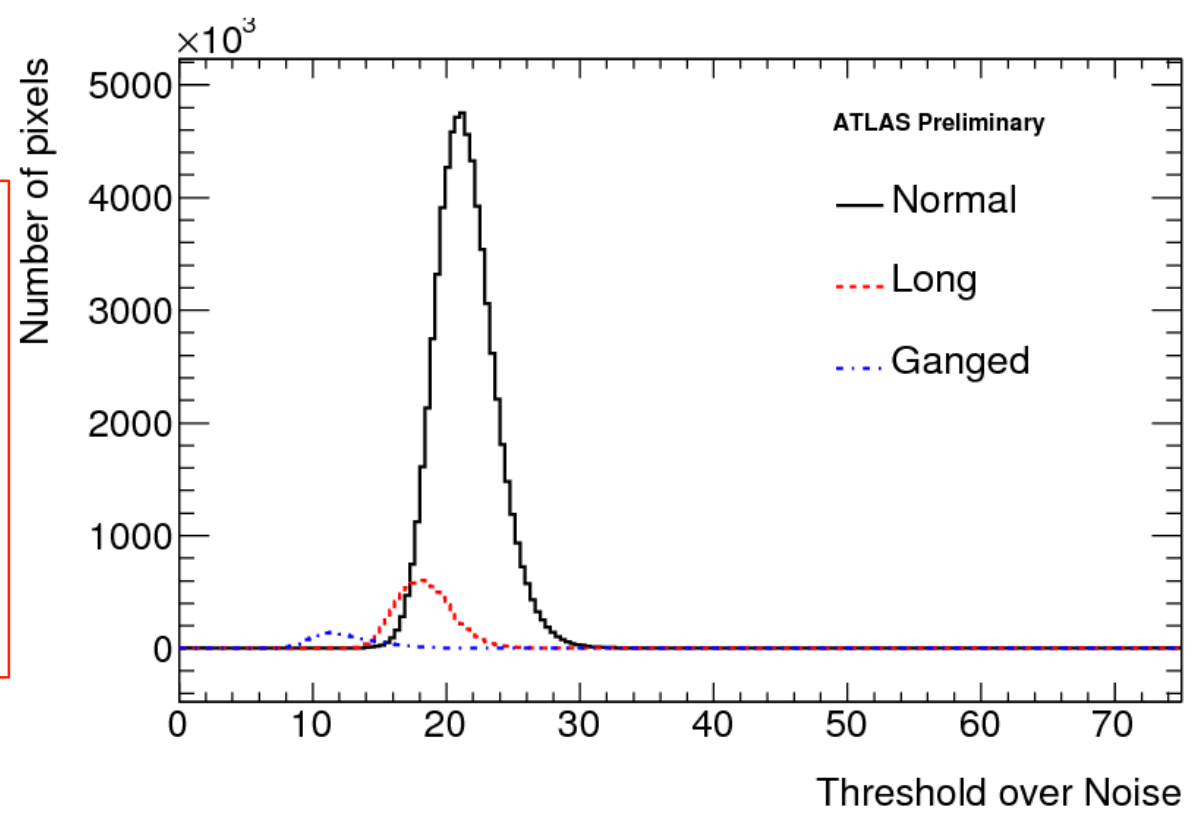




\section{ToT tuning}

Time during which signal is above threshold

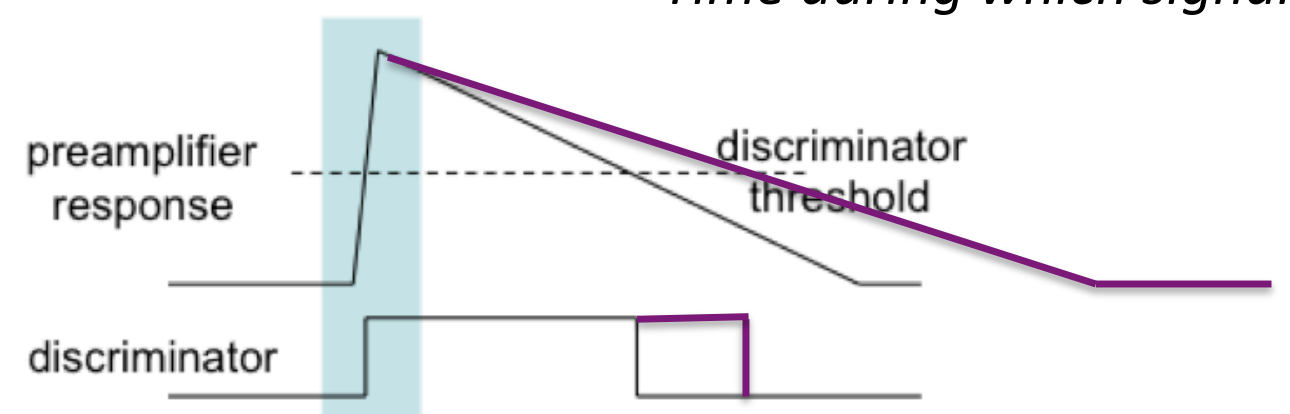

Feedback current dependence

(lower feedback $\rightarrow$ longer ToT)

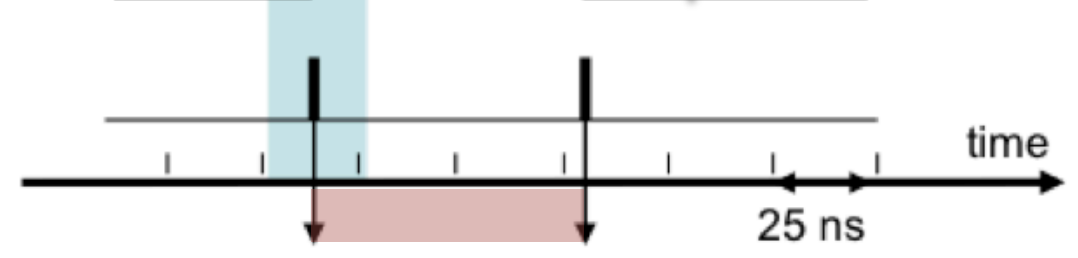

Time over threshold

- In units of Bunch crossing (BC, $25 \mathrm{~ns}$ )

- ToT $\alpha$ deposited charge

- Tuning target : ToT = $30 \mathrm{BC}$ for a m.i.p. (20 ke)

- Resolution : 1BC

\section{Procedure :}

- IFDAC : tune range in which FDAC (feedback current) will be tuned for each pixel

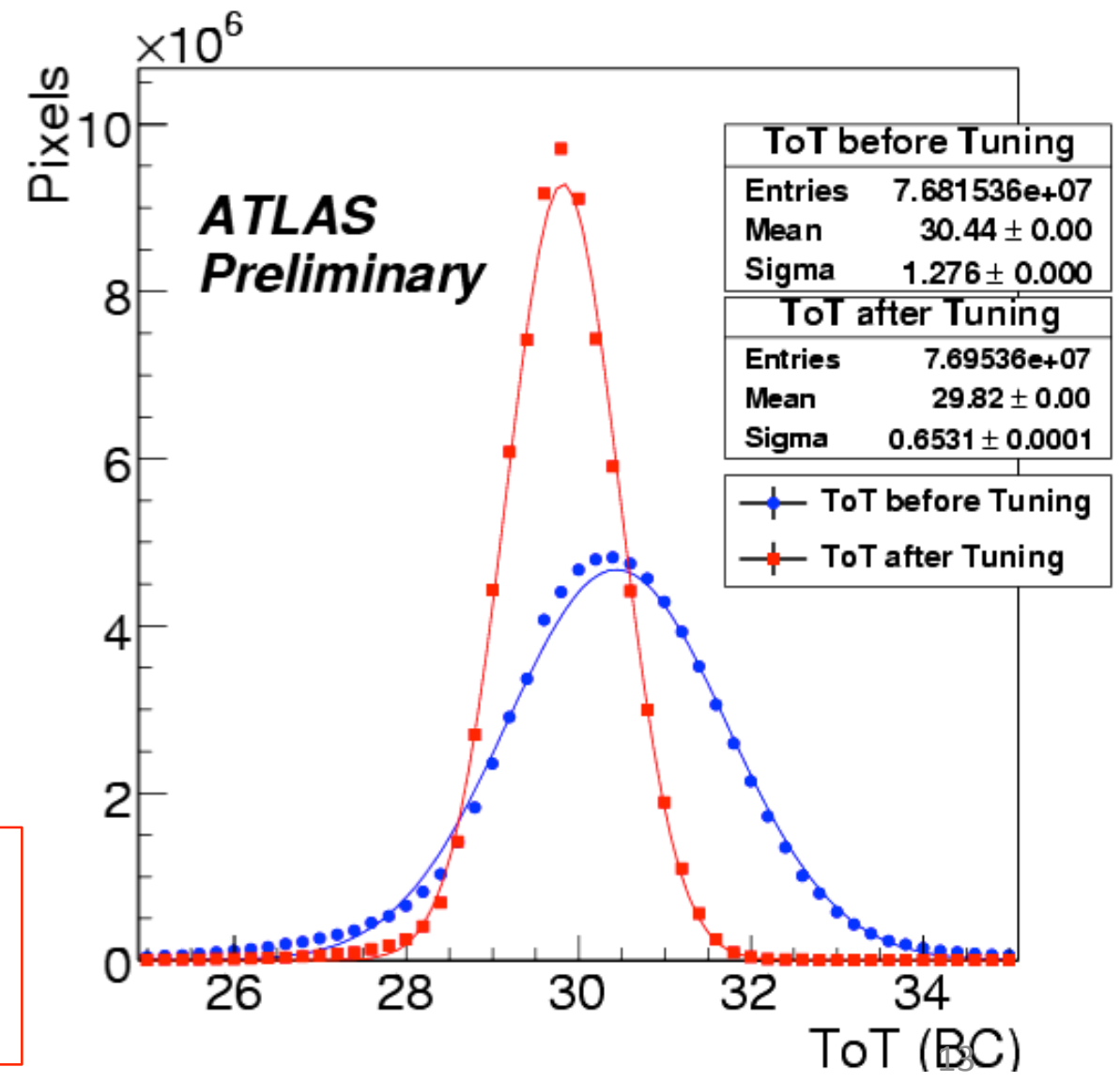




\section{ToT calibration}

- After tuning : calibration : ToT vs deposited charge :

- Inject varying charges to measure ToT per pixel vs injected charge.

- Parameterized with ToT $=p 0(p 1+Q) /(p 2+Q)$ for each front-end and each pixel type

- Used by offline $\rightarrow$ better resolution on position with charge sharing algorithm
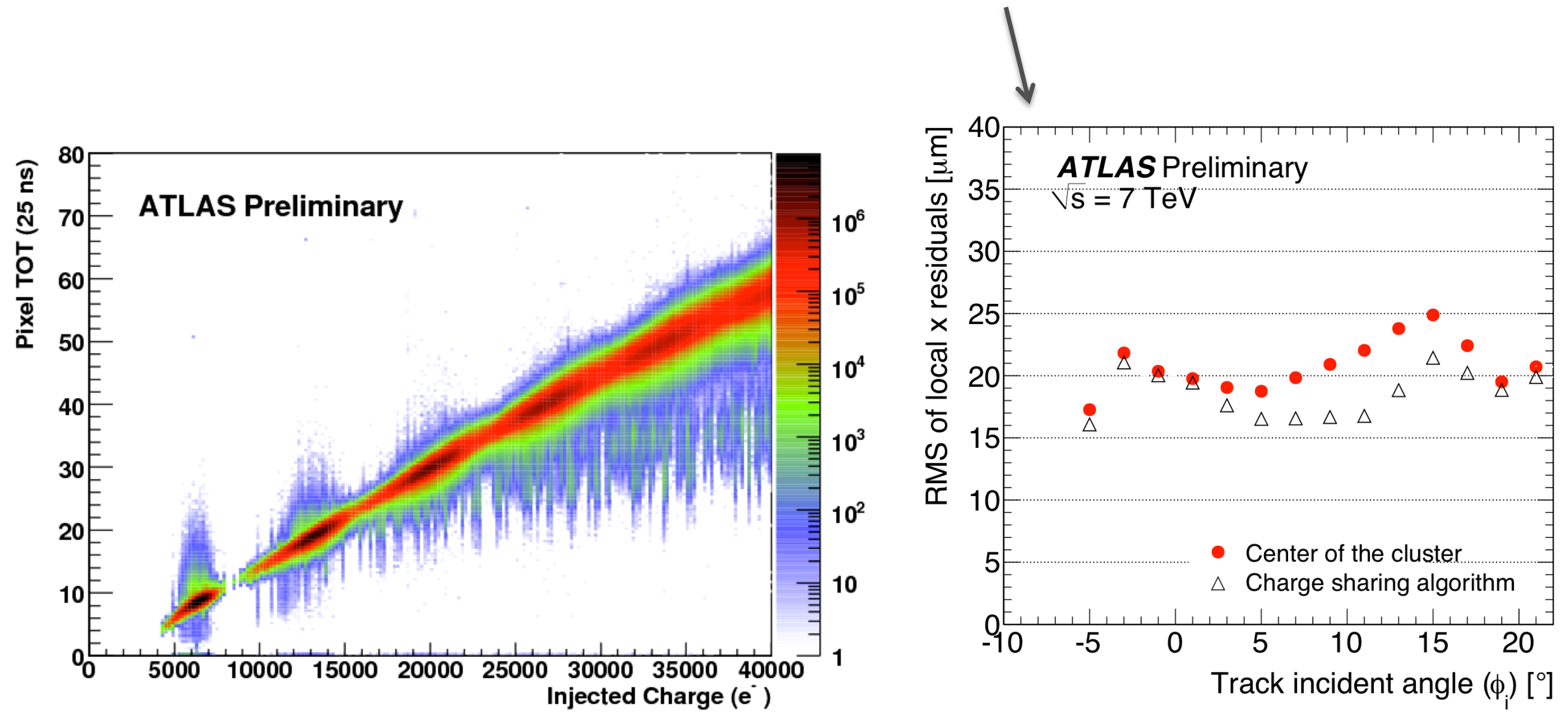


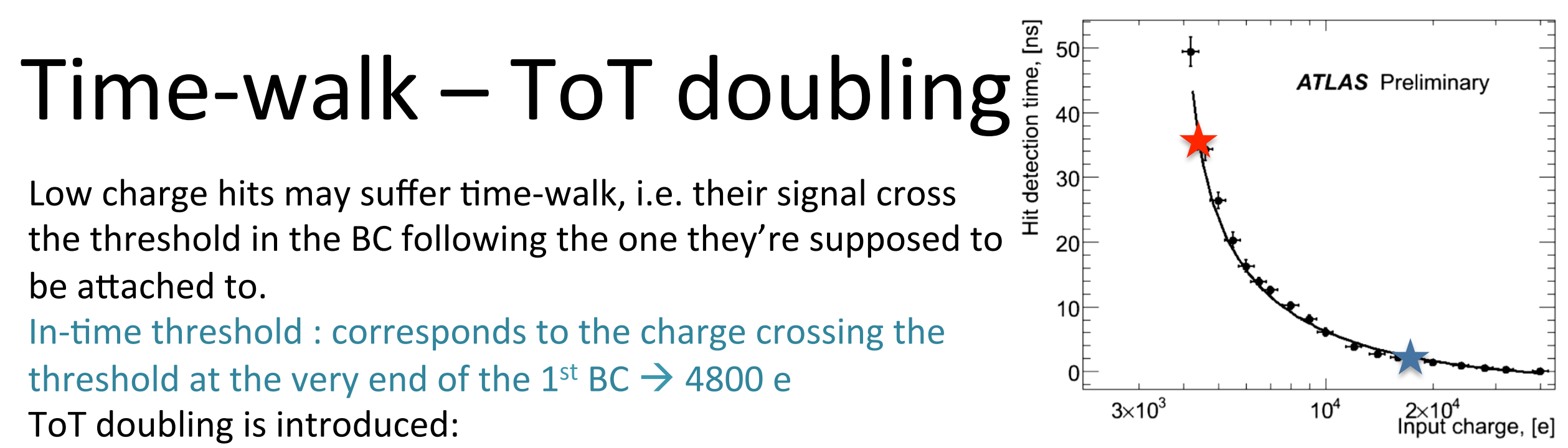

- in-time threshold : 3700 e

All charges measured in the $2^{\text {nd }} \mathrm{BC}$ with a ToT smaller than $\frac{\infty}{\mathbb{0}} 14$ the one corresponding to a 3700 e ( 7BC) charge will be duplicated into the $1^{\text {st }} \mathrm{BC}$

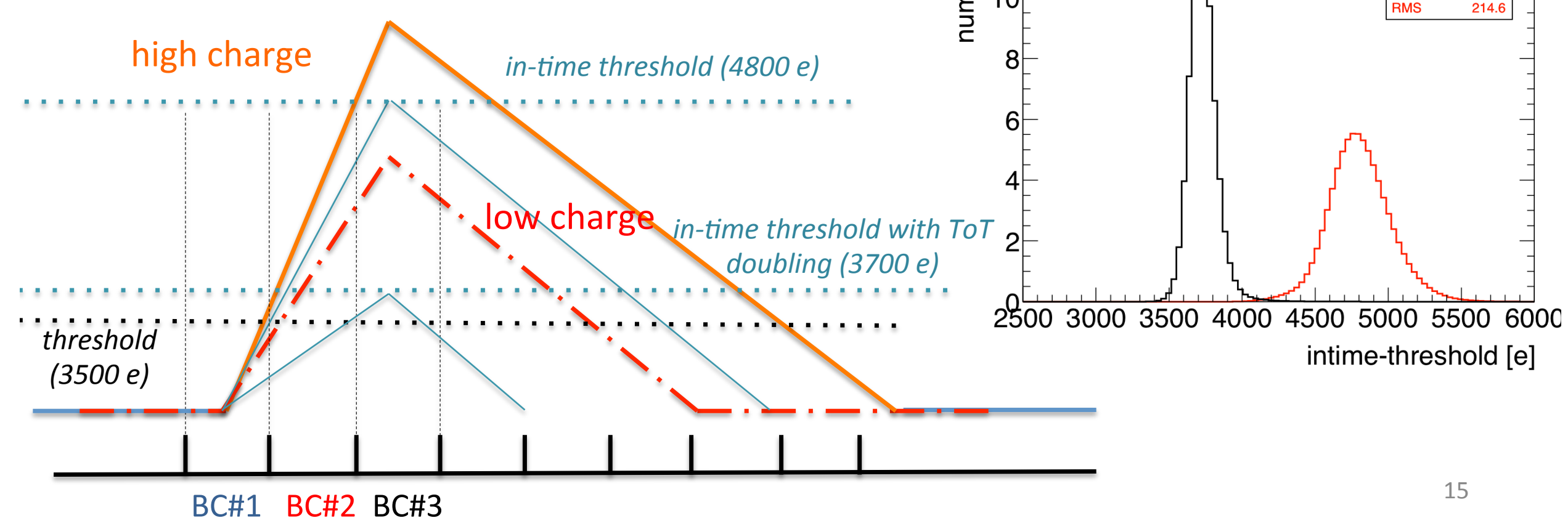




\section{Noise}

- Without noise masking : 1 noise hit/event on the whole Pixel Detector.

- During Pixel Detector standalone runs, noisy pixels (occupancy $>10^{-6}$ ) are identified and masked online

- $\sim 0.1 \%$ for a 3500 e threshold tuning

- Noisy pixel mask then transmitted to offline - additional ones masked offline on run-byrun analysis

- Noise rate after offline masking : $<10^{-9}$

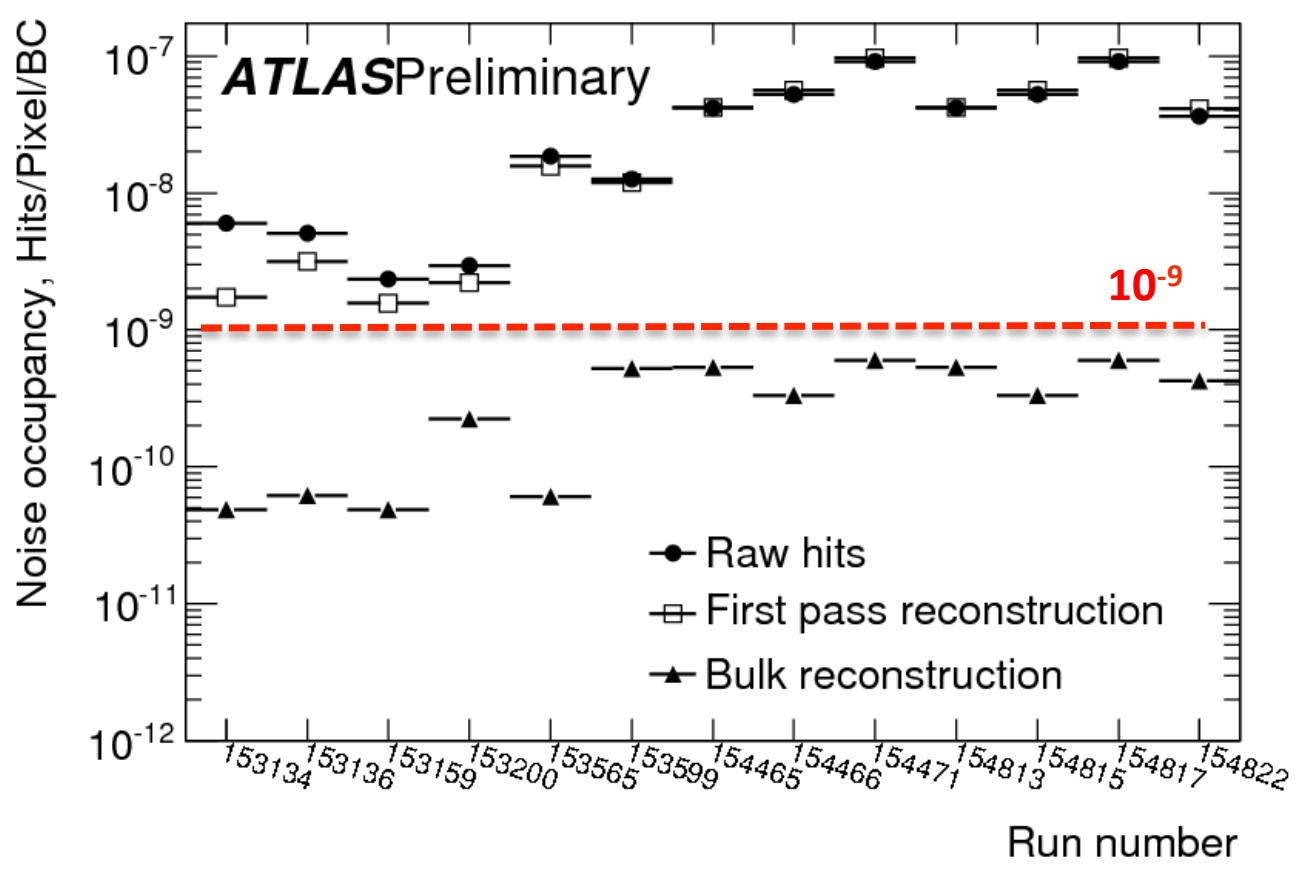




\section{Hit-to-track association efficiency}

- Track selection requirement :

- 1 hit on b-layer ( $\rightarrow 100 \%$ efficient)

- Disabled modules taken into account

- Disabled FE or dead pixels not (main reason for efficiency decrease in outermost discs)

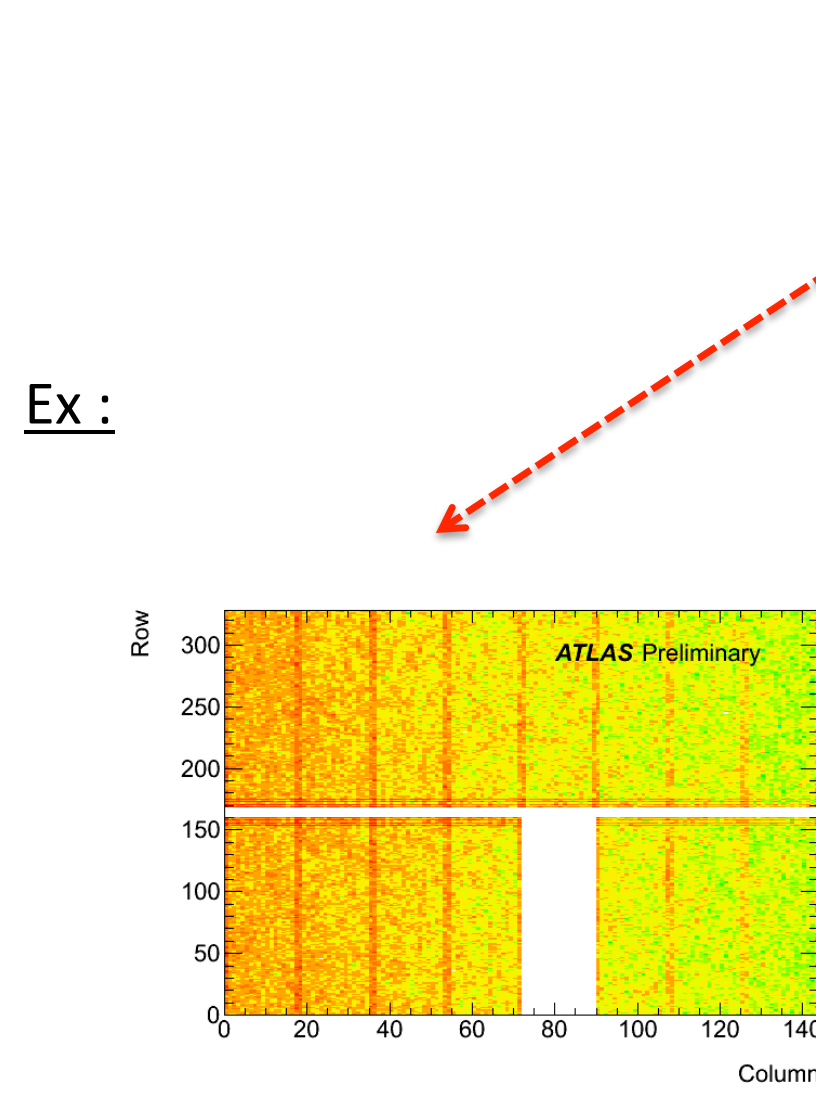




\section{Damages}

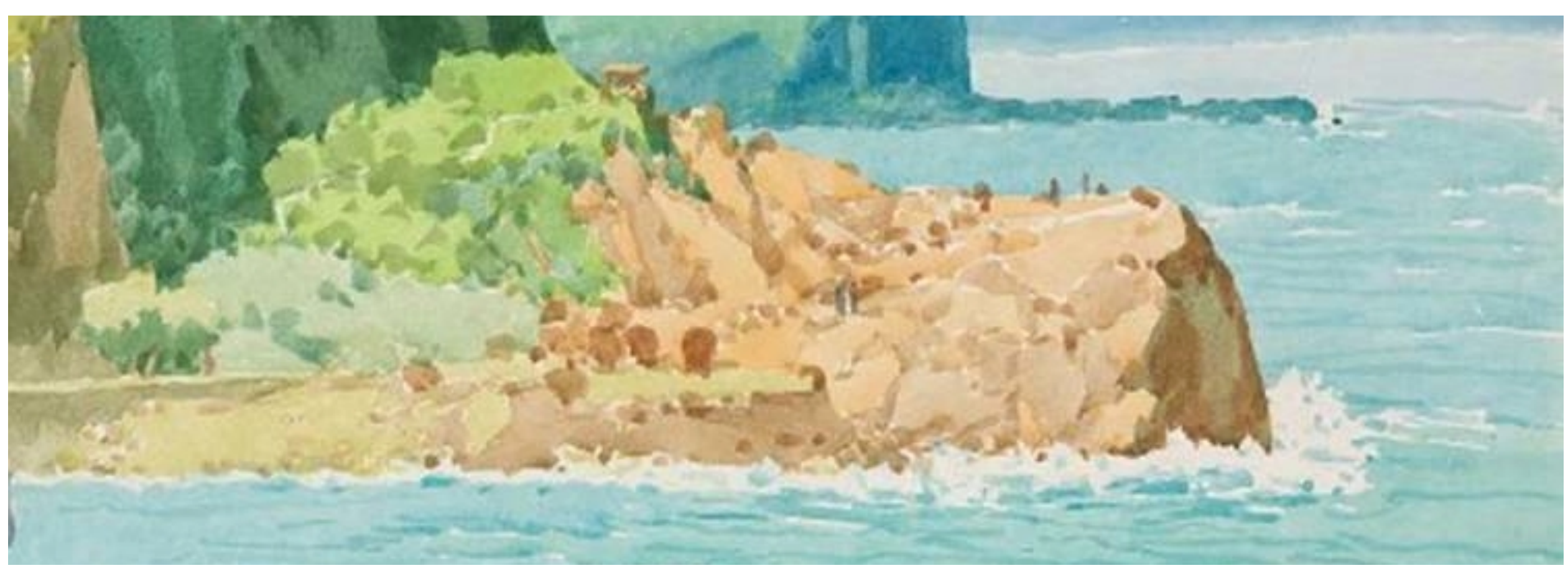




\section{Non-working modules}

- On the $14 / 11 / 11$ :

- 64 dead modules ( 6 due to a single optoboard failure)

- 9 on disks (4 on A side, 5 on $C$ side)

- 9 on Layer 0 (3 central modules)

- 7 on Layer 1

- 39 on Layer 2

- 48 disabled front-ends

- $\rightarrow 3.7 \%$ of the detector disabled (3.8\% if taking into account the disabled FEs)

- $0.1 \%$ of pixels have disconnected bumps (most of them on a couple of disk modules)

- $0.04 \%$ are analog dead $-10 \%$ of them have merged bumps

The percentage of disabled modules grew from $2.1 \%$ to $3.8 \%$ in 3.5 years of operations $\rightarrow$ IBL needed to restore the initial performance.

Inactive fraction per layer: Disabled modules by failure type:

$\begin{array}{ll}\text { B-layer } & 3.1 \% \\ \text { Layer 1 } & 1.4 \% \\ \text { Layer 2 } & 5.7 \% \\ \text { Endcap A } & 2.8 \% \\ \text { Endcap C } & 3.5 \%\end{array}$

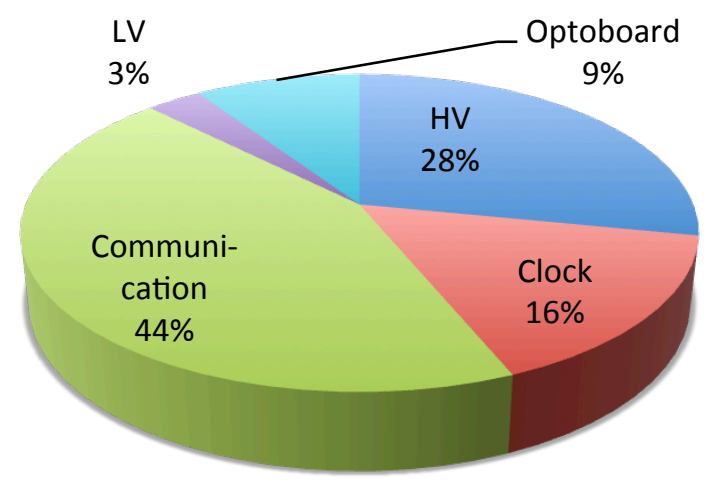




\section{Radiation damages}

- Effects of radiation on pixels :

- Increase of leakage current

- Decrease of voltage for full depletion of sensors until type inversion* (then increase)

- Decrease in charge collection efficiency (lower threshold needed)

- Leakage current :

- Leakage current increase already measurable at the module level but only on the blayer at pixel level
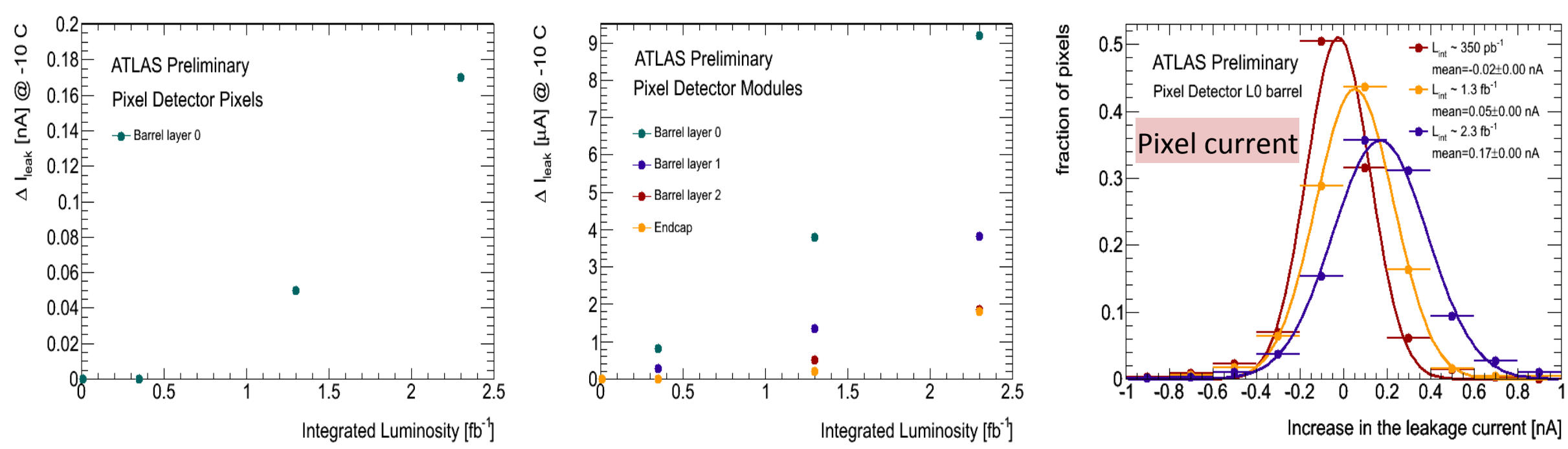

- Increase of $0.17 \mathrm{nA}$ for $2.3 \mathrm{fb}^{-1}$ integrated luminosity for the innermost layer and observation of radial dependence for the endcap modules (pixel level)

*The b-layer is expected to undergo type inversion after $10 \mathrm{fb}^{-1}$ integrated luminosity 


\section{Radiation damages}

- Depletion voltage :

- Shows damage increase

- Indicator of time of type inversion

- During calibration periods and before type inversion, using cross-talk scans :

- Injecting charge in a given pixel - reading out neighbors

- If not fully depleted : high-ohmic short between pixels (cross-talk).

- If fully depleted : pixels are isolated from each other - no hits.

Module close to depletion voltage ->

white already fully depleted (no crosstalk hits). Structure appearing shows the sensor production (Cristal polishing)

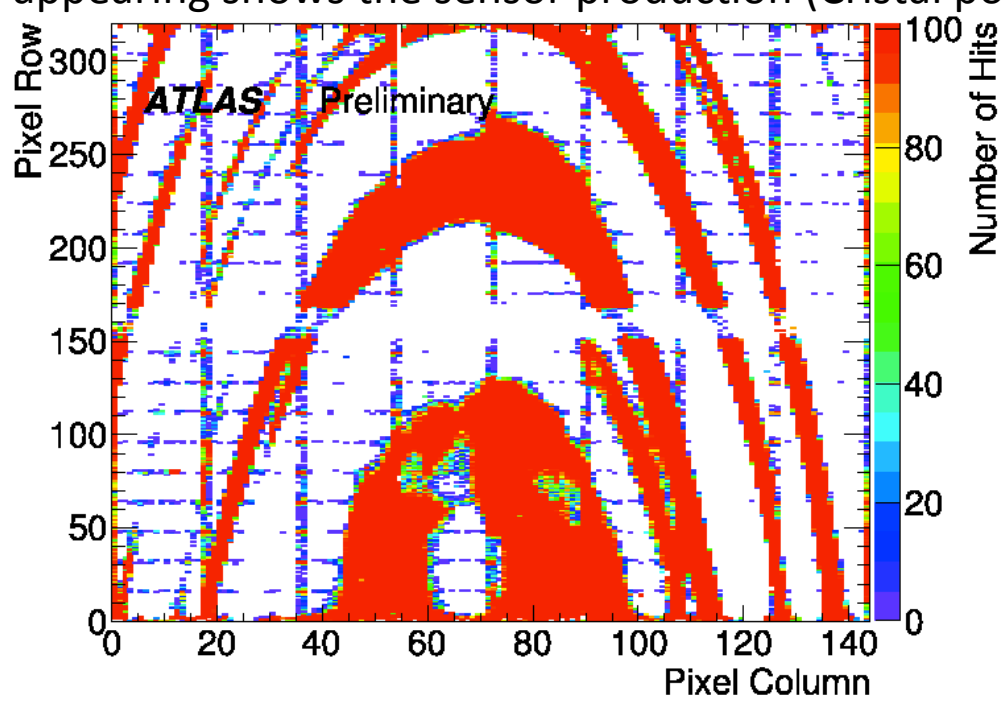

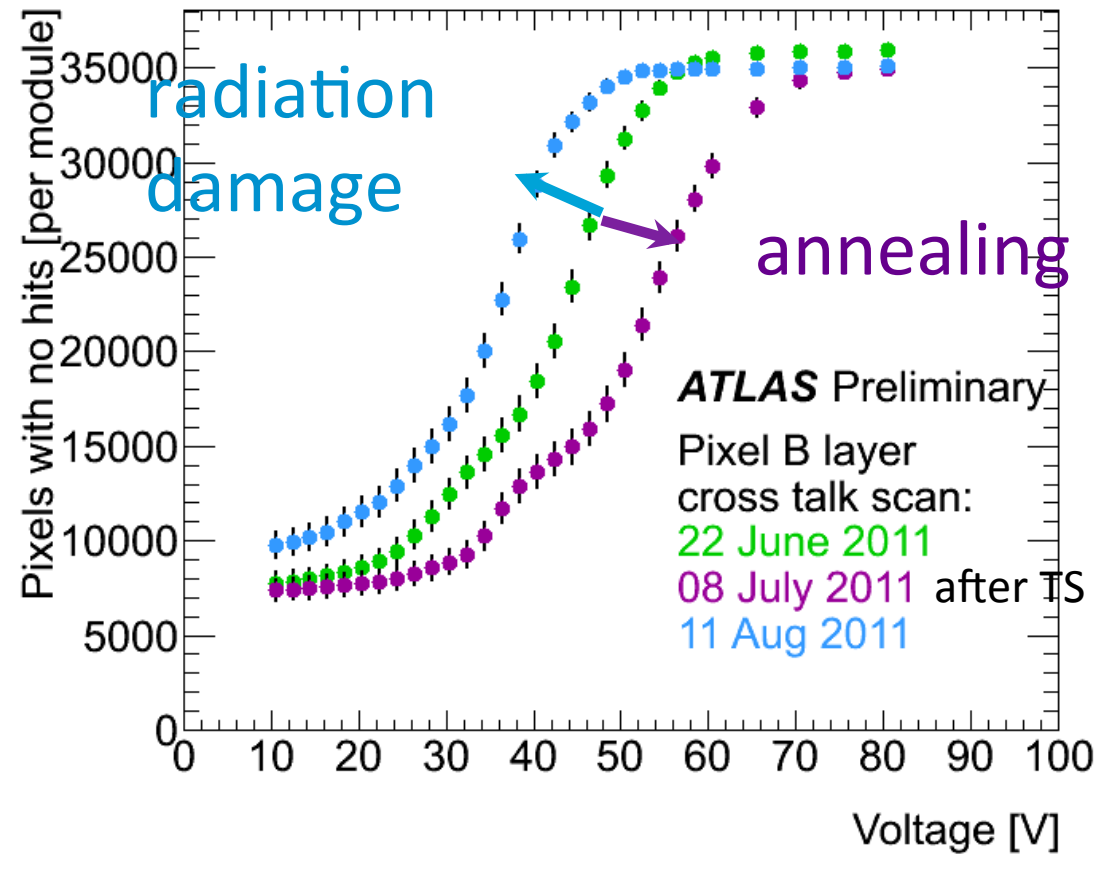




\section{Summary}

\begin{tabular}{|c|c|c|c|c|c|c|c|c|c|c|c|c|}
\hline \multicolumn{3}{|c|}{$\begin{array}{l}\text { Inner Tracking } \\
\text { Detectors }\end{array}$} & \multicolumn{4}{|c|}{ Calorimeters } & \multicolumn{4}{|c|}{ Muon Detectors } & \multicolumn{2}{|c|}{ Magnets } \\
\hline Pixel & SCT & TRT & $\begin{array}{l}\text { LAr } \\
\text { EM }\end{array}$ & $\begin{array}{l}\text { LAr } \\
\text { HAD }\end{array}$ & $\begin{array}{c}\text { LAr } \\
\text { FWD }\end{array}$ & Tile & MDT & $\mathrm{RPC}$ & $\mathrm{CSC}$ & TGC & Solenoid & Toroid \\
\hline 99.8 & 99.6 & 99.2 & 97.5 & 99.2 & 99.5 & 99.2 & 99.4 & 98.8 & 99.4 & 99.1 & 99.8 & 99.3 \\
\hline
\end{tabular}

- Calibrated and very well-performing detector

- Threshold tuned at 3500 e with a dispersion of 40 e, average noise $\sim 170 \mathrm{e}$

- ToT tuned to $30 \mathrm{BC}$ with a resolution of $1 \mathrm{BC}$

- $96.3 \%$ of the detector operational

- offline noise rate $<10^{-9}$

- Data taking efficiency $>99 \%$

- Hit-to-track association efficiency 99\%

- Regularly checked and recalibrated

- Radiation damage : observable but pixels can stand for about 100 times more radiation

- In 2014, thanks to IBL, performance restored and improved 


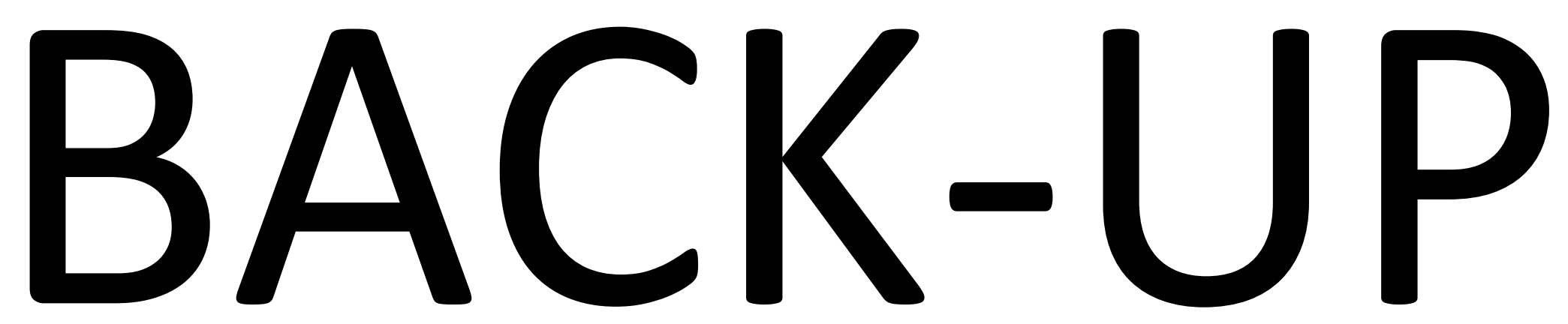




\section{Threshold}
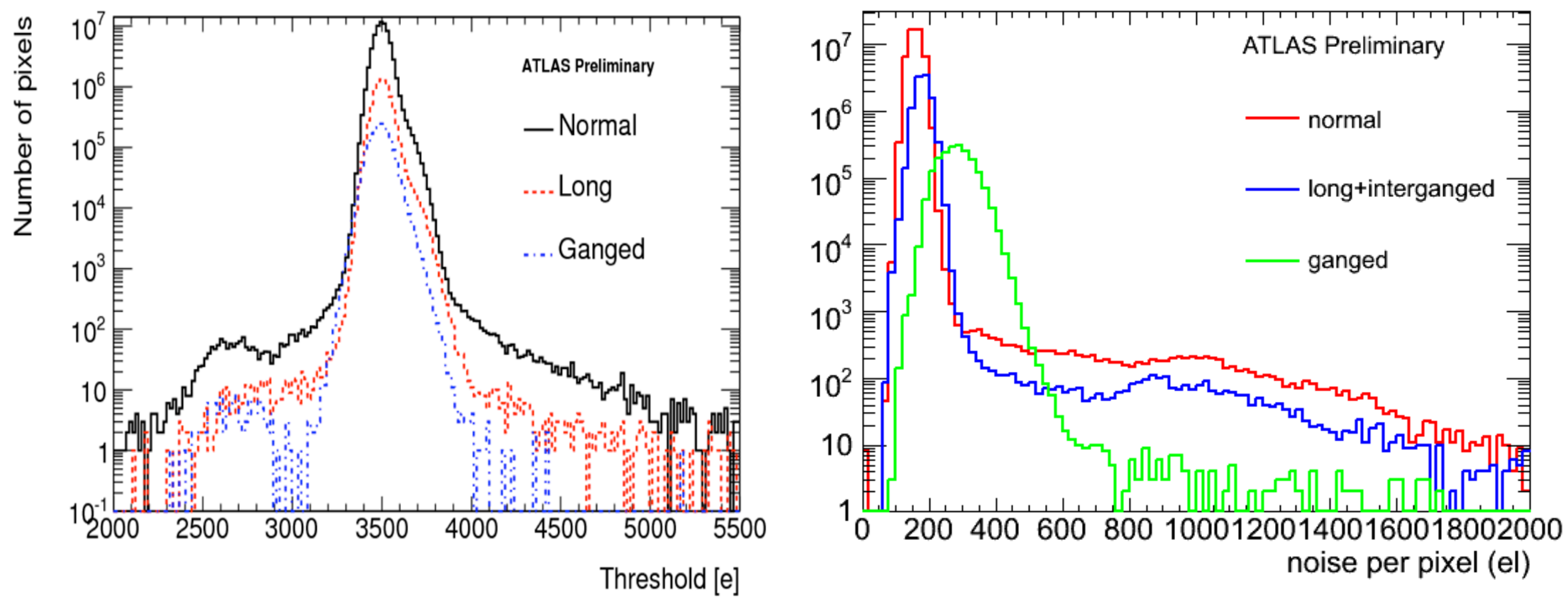


\section{Timing adjustment}

trigger delays $\rightarrow$ done using cosmic ray data cable length $\rightarrow$ measured during installation module-by-module variations

After all adjustment : module-to-module dispersion : $0.007 \mathrm{BC} \rightarrow$ corresponds to a dispersion of $0.17 n$ s (between modules)

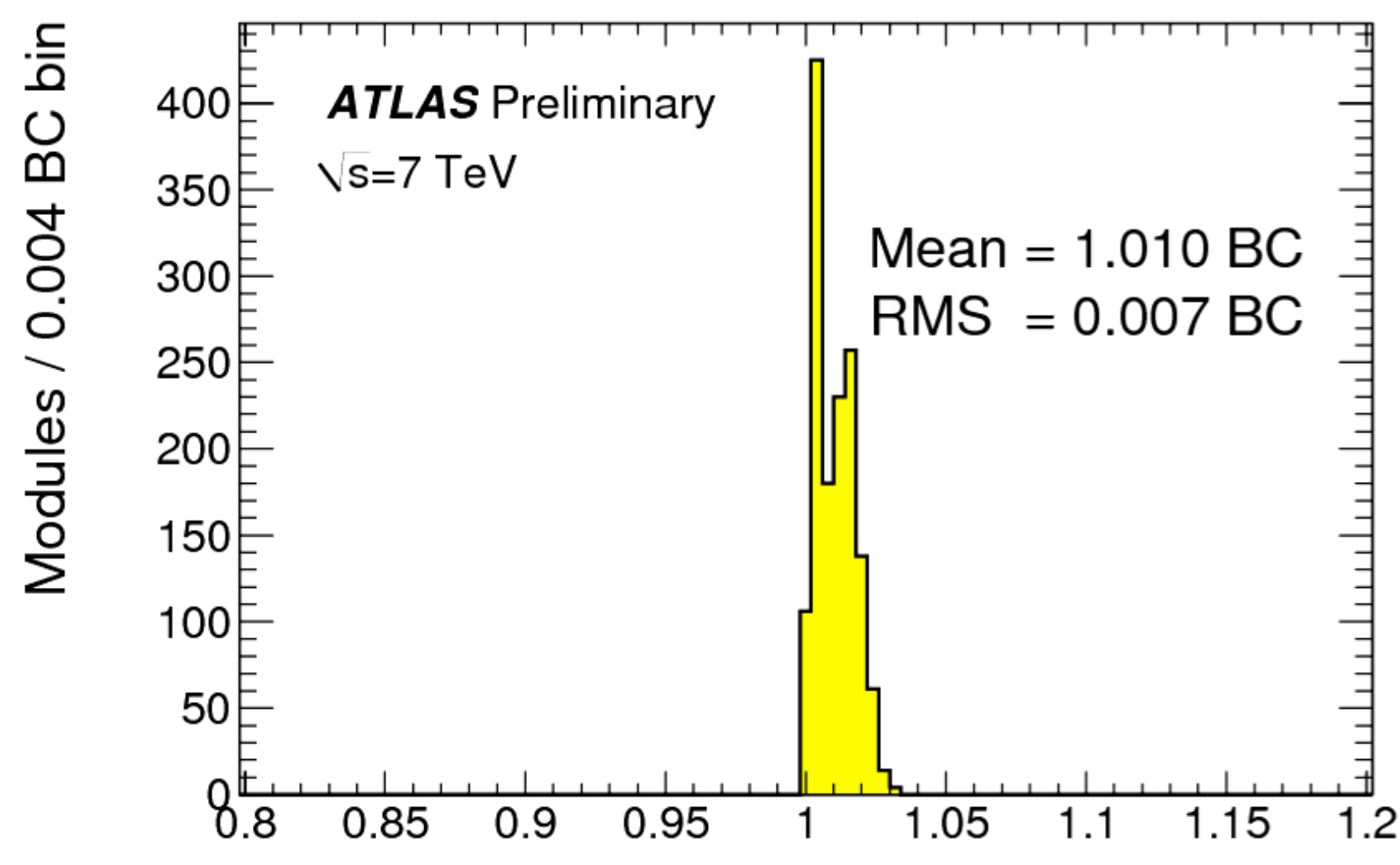

Module $<\mathrm{BC}>[\mathrm{BC}]$ 


\section{Lorentz angle}

- Cluster size vs. track angle with and without magnetic field $\rightarrow$ Measurement of the Lorentz angle

- Measured value of $~ 211 \mathrm{mrad}$, close to expected value (225 mrad)

- Theoretically-expected dependence on mobility can be nicely seen when looking at modules of different temperature

- Measured: $(-0.78 \pm 0.18) \mathrm{mrad} / \mathrm{K}$, expected: $-0.74 \mathrm{mrad} / \mathrm{K}$
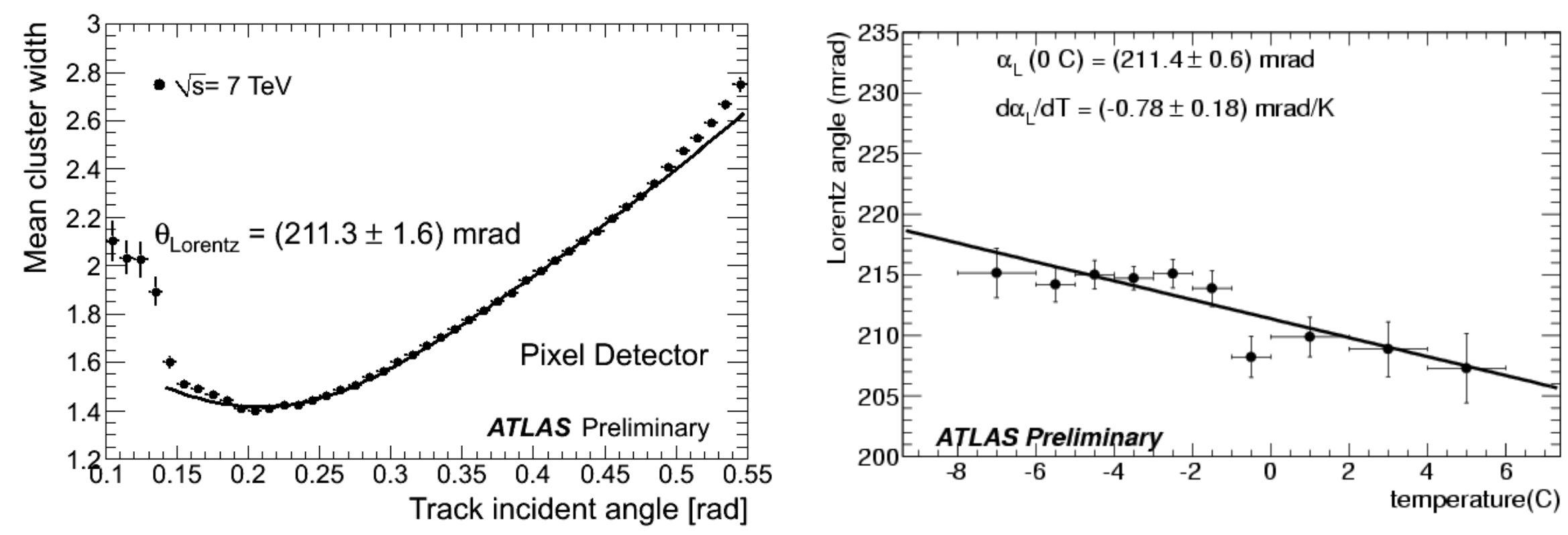


\section{$\mathrm{dE} / \mathrm{dx}$ - particle ID}

track $\mathrm{dE} / \mathrm{dx}$ resolution : $12 \%$

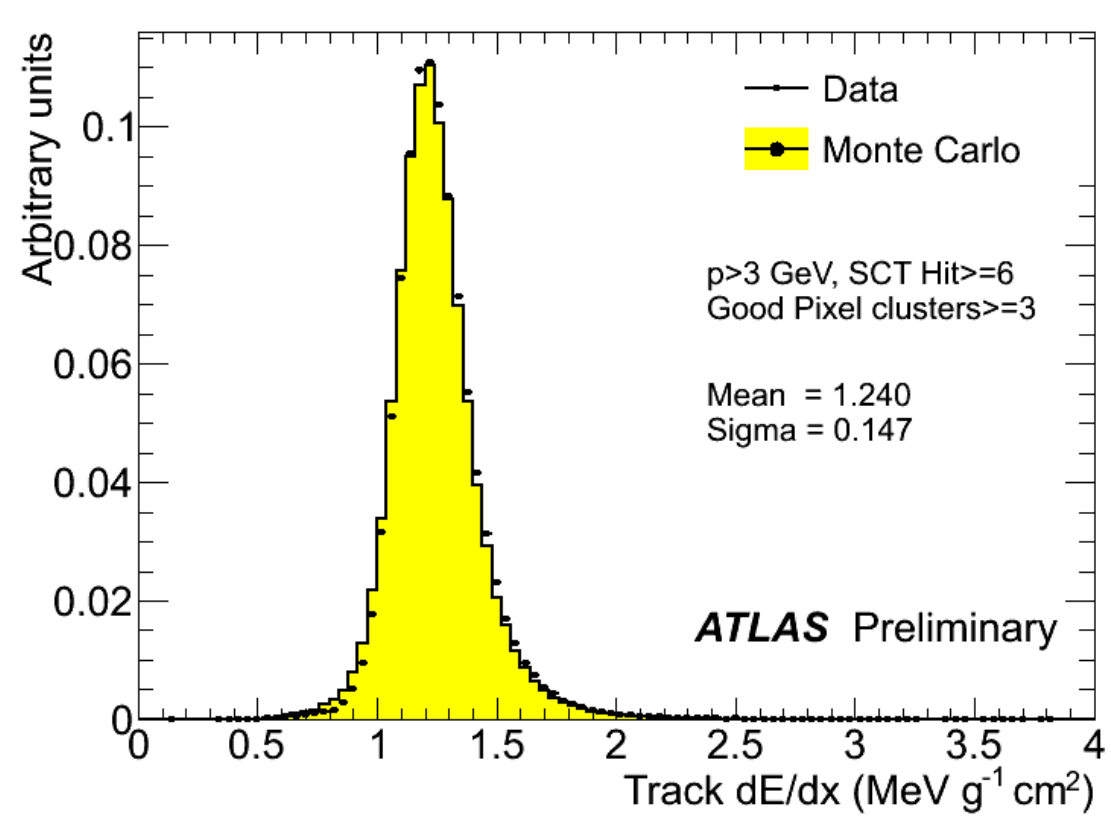

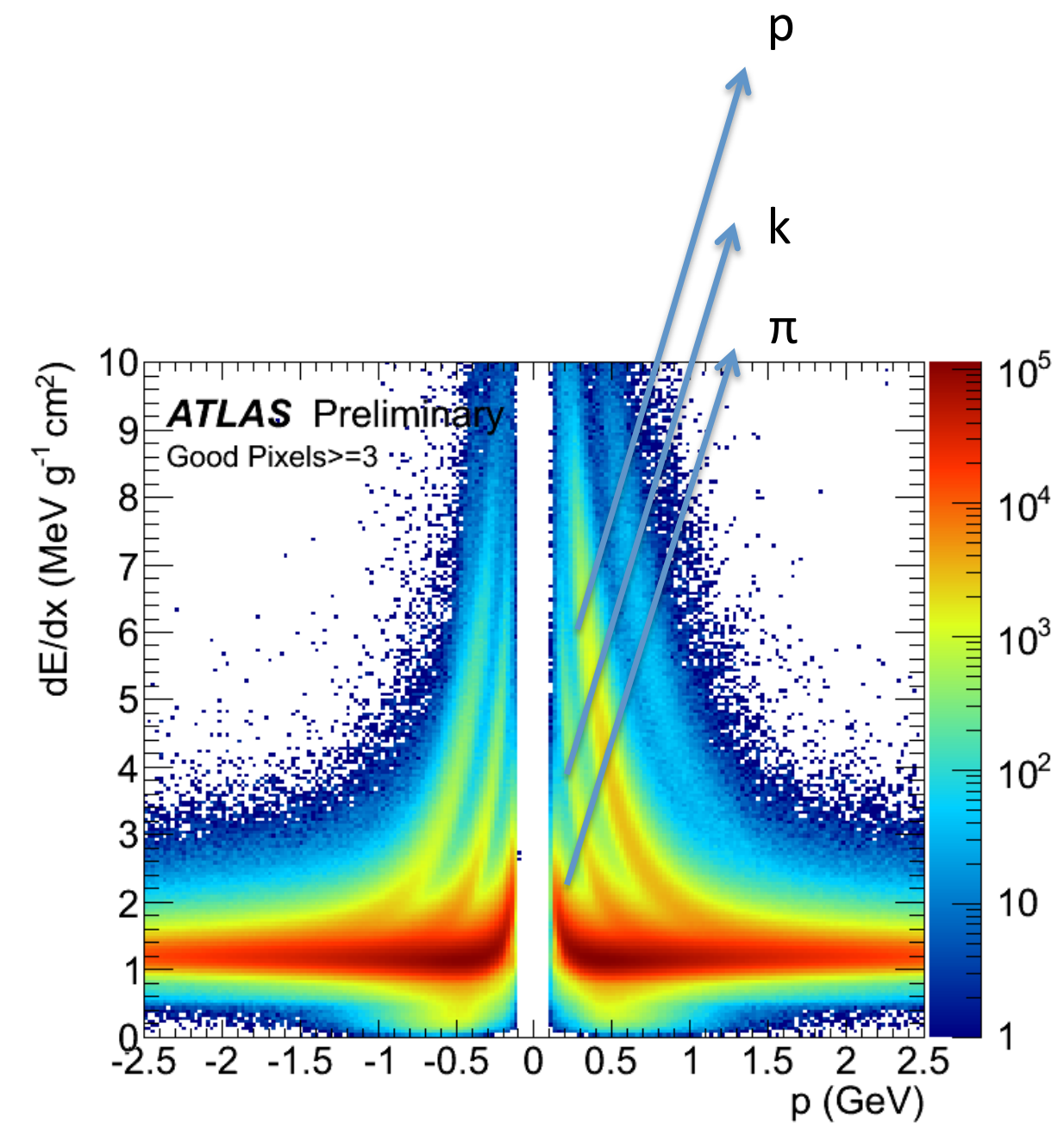




\section{Leakage current}

Radial dependence for the endcap modules (pixel level)
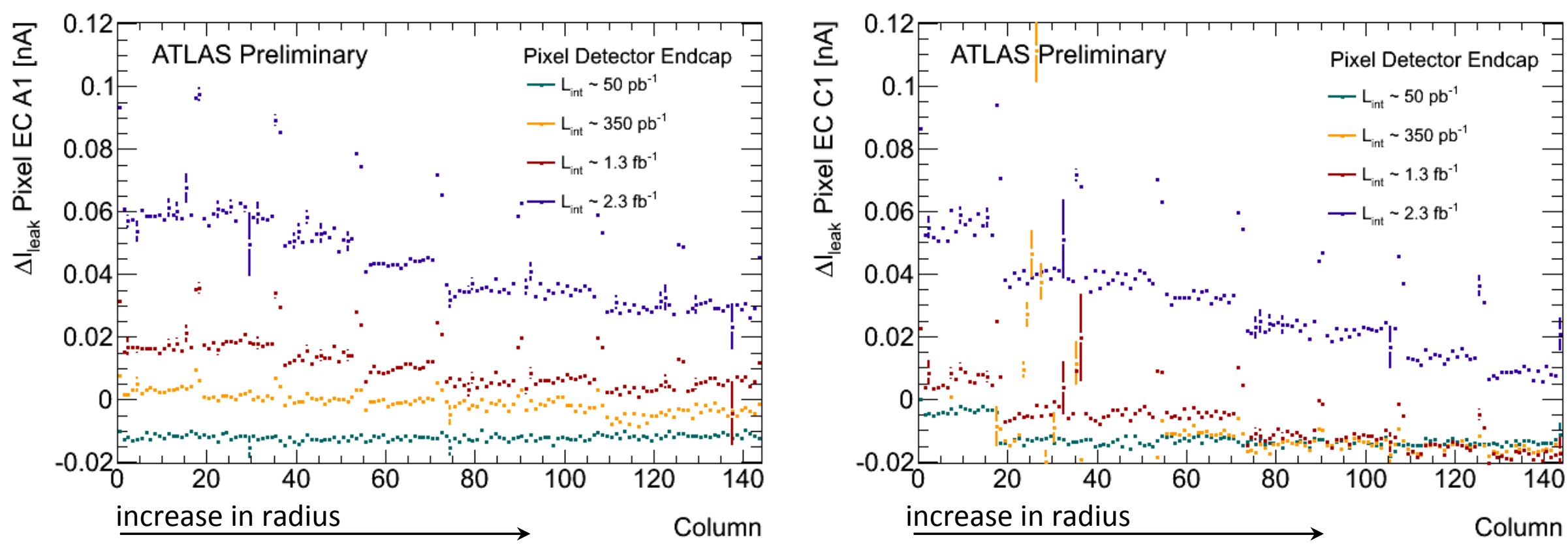